\title{
Orthostatic Blood Pressure Recovery Measured Using a Sphygmomanometer Is Not Associated with Physical Performance or Number of Falls in Geriatric Outpatients
}

\author{
Arjen Mol ${ }^{\mathrm{a}, \mathrm{b}}$ Marieke Esmé Charlotte Blom ${ }^{\mathrm{a}}$ Danique Johanna van den Bosch ${ }^{\mathrm{a}}$ \\ Richard Jack Anton Van Wezel ${ }^{b, c}$ Carel G.M. Meskers ${ }^{d}$ Andrea B. Maier ${ }^{a, e}, f, g$ \\ aDepartment of Human Movement Sciences, @AgeAmsterdam, Amsterdam Movement Sciences, Vrije Universiteit, \\ Amsterdam, The Netherlands; ' Department of Biophysics, Donders Institute for Brain, Cognition and Behaviour, \\ Radboud University, Nijmegen, The Netherlands; 'Biomedical Signals and Systems, MIRA Institute for Biomedical \\ Technology and Technical Medicine, University of Twente, Enschede, The Netherlands; ${ }^{\mathrm{d} D e p a r t m e n t}$ of \\ Rehabilitation Medicine, Amsterdam University Medical Center, Amsterdam Movement Sciences, Amsterdam,

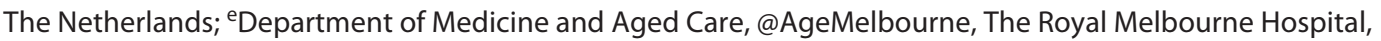 \\ The University of Melbourne, Melbourne, VIC, Australia; ${ }^{f}$ Healthy Longevity Translational Research Program, Yong \\ Loo Lin School of Medicine, National University of Singapore, Singapore, Singapore; ${ }^{9}$ Centre for Healthy Longevity, \\ @AgeSingapore, National University Health System, Singapore, Singapore
}

\section{Keywords}

Orthostatic hypotension - Chair stand test .

Sphygmomanometer

\begin{abstract}
Background: Orthostatic hypotension $(\mathrm{OH})$ and impaired $\mathrm{OH}$ recovery derived from beat-to-beat blood pressure (BP) measurements are associated with detrimental clinical outcome, but the clinical relevance of $\mathrm{OH}$ recovery assessed using the widely available sphygmomanometer is still unclear. Method: 635 geriatric outpatients underwent comprehensive geriatric assessment, including orthostatic BP measurements using a sphygmomanometer, during supine rest and 1 and 3 min after standing up and assessment of physical performance (i.e., the timed up and go test and the Short Physical Performance Battery) and the number of falls in the past year. The association between BP recovery, defined as $\mathrm{BP}$ at 3 min minus BP at 1 min after standing up, with physical performance and falls was assessed using regression analyses, adjusting for age and sex, both in the entire cohort and after stratifying for the presence of $\mathrm{OH}$ at 1 min after
\end{abstract}

karger@karger.com www.karger.com/ger

Karger!

BOPEN ACCESS
C 2021 The Author(s).

Published by S. Karger AG, Basel

This is an Open Access article licensed under the Creative Commons Attribution-NonCommercial-4.0 International License (CC BY-NC) (http://www.karger.com/Services/OpenAccessLicense), applicable to the online version of the article only. Usage and distribution for commercial purposes requires written permission. standing up. Results: BP recovery was not associated with physical performance or number of falls, neither in the entire cohort, nor in subpopulations with or without $\mathrm{OH}$. Conclusion: The clinical relevance of BP recovery between 1 and 3 min after standing up could not be demonstrated. The results suggest that sphygmomanometer measurements have an inadequate time resolution to record the clinically relevant dynamics of orthostatic BP recovery.

(c) 2021 The Author(s).

Published by S. Karger AG, Basel

\section{Introduction}

Orthostatic hypotension $(\mathrm{OH})$ is a systolic/diastolic blood pressure (SBP/DBP) of $>20 / 10 \mathrm{~mm} \mathrm{Hg}$ within 3 min after standing up [1]. OH may be accompanied by symptoms of dizziness, fainting, and falls and is associated with impaired physical and cognitive functioning, falls, morbidity, and mortality [2-7]. Impaired recovery

M.E.C. Blom and D.J. van den Bosch contributed equally to this study.
Correspondence to:

Andrea B. Maier, a.b.maier@vu.nl 
from $\mathrm{OH}$ may lead to detrimental clinical outcome by prolonged brain hypoperfusion [2].

Previous studies used a number of different BP recovery measures derived from continuous (beat-to-beat) BP measurements during and after standing up and found a lower BP recovery to be associated with physical performance, frailty, falls, and cognitive decline [8-11] but not with mortality [12-14]. However, continuous BP measurements are most often not available in clinical practice, in contrast to intermittent (sphygmomanometer) BP measurements. Studies on the relationship between timing of intermittent orthostatic BP measurements and clinical outcome are contradictory $[15,16]$, and no studies have addressed the association between intermittently assessed BP recovery and clinical outcome. Intermittently measured BP recovery between 1 and 3 min after standing up may reflect the effectiveness of the baroreflex, arterial and venous vasoconstriction, and cardiac contractility [9].

This study assessed the association between orthostatic BP recovery between 1 and 3 min measured using a sphygmomanometer and physical performance and falls in geriatric outpatients. We hypothesized that a higher BP recovery is associated with better physical performance and a lower incidence of falls.

\section{Methods}

Study Population and Design

Patients referred to the geriatric outpatient clinic of Bronovo hospital (The Hague, The Netherlands; March 2011 to January 2012; $N=257$ ) and the Center of Geriatrics Amsterdam (Amsterdam UMC, The Netherlands; January 2014 to December 2015; $N=378$ ) undergoing a comprehensive geriatric assessment were included in this study.

\section{Data Collection}

Information about age, sex, height, weight, living situation, and smoking habits were extracted from medical records. BP measurements were performed in all patients in the supine position (baseline) and after 1 and 3 min standing up using a sphygmomanometer. Patients measured in the Bronovo hospital were supported with an automatic lift chair (Vario 570; Fitform B.V., Best, The Netherlands) during standing up.

Physical performance was assessed according to the timed up and go test (TUG) and Short Physical Performance Battery (SPPB). The TUG measures the time needed to stand up from a chair without using the hands, walk around a cone, and be seated again [17]. The SPPB tests the capacity to maintain balance during $10 \mathrm{~s}$ of side-by-side stance, semi-tandem stance and tandem stance, preferred walking speed on a 4- $m$ trajectory, and the time needed to complete the chair stand test (CST) [18]. The CST measures the time needed to stand up from a chair and sit down for 5 times as quickly as possible [18]. The number of falls was assessed using a questionnaire asking for the number of falls in the past year. All measurements and the questionnaire were per- formed during the same visit as part of the comprehensive geriatric assessment, in the following order: falls questionnaire, orthostatic BP, CST, walking speed, and TUG measurements.

\section{Data Analysis}

All analyses were performed using IBM SPSS Statistics, version 25. Data are presented using mean and standard deviation in case of normal distribution and using median and interquartile range (IQR) otherwise.

$\mathrm{OH}$ was defined as a systolic drop of at least $20 \mathrm{~mm} \mathrm{Hg}$ and/or a diastolic drop of at least $10 \mathrm{~mm} \mathrm{Hg} \mathrm{BP}$ relative to baseline at either 1 or 3 min after standing up. BP recovery was defined as BP measured at 3 min after standing up minus BP measured at 1 min after standing up.

Prior to regression analysis, log transformations were applied to the CST and TUG times to obtain normal distributions. SPPB total score and number of falls were dichotomized at their median value. SPPB total score was also divided into categories: disabled (score 0-2), frail (score 3-9), and nonfrail (score 10-12).

The association between BP recovery and TUG, $4 \mathrm{mWS}$, and CST was assessed using linear regression, adjusting for age and sex. The association between BP recovery and SPPB total score and number of falls was assessed using logistic regression, adjusting for age and sex. Linear regression models could not be used in this analysis because of the nonnormal distribution of the number of falls. All analyses were performed both in the entire group of patients and after stratifying for the presence of $\mathrm{OH}$ at $1 \mathrm{~min}$ after standing up. Statistical significance was set at 0.05 and Bonferroni corrected for multiple (ten) comparisons.

\section{Results}

Orthostatic BP, physical performance, and falls data were available for 635 geriatric patients. The patient characteristics are presented in Table 1 . The mean age of patients was 81.1 (standard deviation 6.9 ) years. $41.1 \%$ of the patients were men and $52.4 \%$ were living independently. Median baseline SBP and DBP were 144 (IQR 132-163) $\mathrm{mm} \mathrm{Hg}$ and 79 (IQR 72-87) $\mathrm{mm} \mathrm{Hg}$, respectively. 179/635 (28.2\%) patients had OH. Median SBP and DBP recovery was 4.0 (IQR -3.0 to 12.0 ) $\mathrm{mm} \mathrm{Hg}$ and 2.0 (IQR -2.0 to 5.0$) \mathrm{mm} \mathrm{Hg}$, respectively.

Table 2 presents the results from the regression analyses on the associations between BP recovery and physical performance and falls. After correction for multiple comparisons, BP recovery was neither associated with physical performance nor number of falls in the entire populations or in the subpopulations with or without $\mathrm{OH}$.

Online suppl. Supplementary Table 1 (see www.karger.com/doi/10.1159/000515658 for all online suppl. material) lists the results from logistic regression analyses on the associations between BP recovery and SPPB total score categories. No significant associations were found after correction for multiple comparisons. 
Table 1. Patient characteristics

\begin{tabular}{|c|c|c|c|c|c|c|}
\hline Characteristic & $N$ & $\begin{array}{l}\text { COGA } \\
(N=378)\end{array}$ & $N$ & $\begin{array}{l}\text { Bronovo } \\
(N=257)\end{array}$ & $N$ & $\begin{array}{l}\text { All } \\
(N=635)\end{array}$ \\
\hline \multicolumn{7}{|l|}{ Sociodemographics } \\
\hline Age, mean (SD), years & 378 & $80.5(6.6)$ & 257 & $82.0(7.2)$ & 635 & $81.1(6.9)$ \\
\hline Male, $n(\%)$ & 378 & $165(43.7)$ & 257 & $96(37.4)$ & 635 & $261(41.1)$ \\
\hline Living independently, $n(\%)$ & 360 & $186(49.2)$ & 246 & $147(57.2)$ & 606 & $333(52.4)$ \\
\hline \multicolumn{7}{|l|}{ Health characteristics } \\
\hline Currently smoking, $n(\%)$ & 350 & $28(7.4)$ & 119 & $22(8.6)$ & 469 & $50(7.9)$ \\
\hline \multicolumn{7}{|l|}{ Supine resting $B P$} \\
\hline SBP, median [IQR], mm Hg & 378 & $146[134-165]$ & 257 & $142[130-157]$ & 635 & $144[132-163]$ \\
\hline DBP, median [IQR], mm Hg & 378 & $81[74-90]$ & 257 & $76.0[69-83]$ & 635 & $79[72-87]$ \\
\hline \multicolumn{7}{|c|}{$O H$ and $B P$ recovery between 1 and 3 min after standing up } \\
\hline $\mathrm{OH},{ }^{1} n(\%)$ & $378^{\circ}$ & $138(36.5)$ & 257 & $41(16.0)$ & 635 & $179(28.2)$ \\
\hline SBP recovery, median $[\mathrm{IQR}], \mathrm{mm} \mathrm{Hg}$ & 378 & $5.0[-2.0$ to 14.0$]$ & 257 & $2.0[-3.0$ to 9.0$]$ & 635 & $4.0[-3.0$ to 12.0$]$ \\
\hline DBP recovery, median $[\mathrm{IQR}], \mathrm{mm} \mathrm{Hg}$ & 378 & $2.0[-2.0$ to 6.0$]$ & 257 & $1.0[-2.0$ to 4.0$]$ & 635 & $2.0[-2.0$ to 5.0$]$ \\
\hline \multicolumn{7}{|l|}{ Physical performance and falls } \\
\hline TUG, median [IQR], s & 265 & $15.0[12.0-18.5]$ & 232 & $16.6[12.6-23.0]$ & 497 & $15.7[12.1-20.4]$ \\
\hline Walking speed, mean (SD)/median [IQR], m/s & 314 & $0.86(0.42)$ & 248 & $0.74[0.55-0.91]$ & 562 & $0.81(0.36)$ \\
\hline CST, median [IQR], s & 304 & $13.9[11.6-18.8]$ & 217 & $15.1[12.3-20.3]$ & 521 & $14.3[11.7-19.4]$ \\
\hline SPPB total score, median [IQR], points & 360 & $8.0[6.0-10.0]$ & 249 & $7.0[5.0-10.0]$ & 609 & $8.0[5.0-10.0]$ \\
\hline SPPB total score $0-2, n(\%)$ & 360 & $19(5.2)$ & 249 & $18(7.2)$ & 609 & $37(6.1)$ \\
\hline SPPB total score $3-9, n(\%)$ & 360 & $216(60.0)$ & 249 & $165(66.3)$ & 609 & $381(62.6)$ \\
\hline SPPB total score $10-12, n(\%)$ & 360 & $125(34.7)$ & 249 & $66(26.5)$ & 609 & $191(31.4)$ \\
\hline Number of falls, median [IQR], counts & 180 & $2.0[1.0-4.0]$ & 157 & $2.0[1.0-4.0]$ & 337 & $2.0[1.0-4.0]$ \\
\hline
\end{tabular}

$\mathrm{OH}$, orthostatic hypotension; SD, standard deviation; IQR, interquartile range; SBP, systolic blood pressure; DBP, diastolic blood pressure; CST, chair stand test; SPPB, Short Physical Performance Battery; TUG, timed up and go test; COGA, Center of Geriatrics Amsterdam. ${ }^{1}$ Number and proportion of patients with $\mathrm{OH}$, defined as a SBP/DBP drop of 20/10 mm $\mathrm{Hg}$ at 1 and/or $3 \mathrm{~min}$ after standing up.

\section{Discussion}

$\mathrm{BP}$ recovery between 1 and 3 min after standing up measured with a sphygmomanometer was not associated with physical performance or number of falls in a large population of geriatric outpatients. The present results point in a different direction than previous studies, which used continuous BP measurements and reported BP recovery between 30 and $60 \mathrm{~s}$ after standing up to be associated with impaired physical performance, frailty, and falls $[9,11]$. Patients may be particularly vulnerable for inadequate BP recovery in this time window as cerebral autoregulation might not have reached its full capacity [19]. Sphygmomanometer BP measurements miss this clinically relevant time window due to their low time resolution. The finding that stratification for the presence of $\mathrm{OH}$ at $1 \mathrm{~min}$ after standing up did not change the results may also be attributed to the fact that the largest BP drop typically occurs within $1 \mathrm{~min}$ and is therefore missed by sphygmomanometer measure- ments [8]. Timing of BP readings at exactly 1 and $3 \mathrm{~min}$ is difficult using a sphygmomanometer due to the required cuff inflation, deflation, and auscultation, potentially resulting in substantial errors. Sphygmomanometer BP measurements do not allow for averaging of BP values within short time intervals (e.g., 5-10 s), which could cancel out random BP fluctuations [9]. These limitations of sphygmomanometer orthostatic BP measurements may explain the different findings between the present study and aforementioned studies. Although physical performance was measured objectively, self-reported falls may be subject to recall bias, which is a limitation of this study.

\section{Conclusions, Implications, and Further Directions}

$\mathrm{BP}$ recovery between 1 and 3 min after standing up measured with a sphygmomanometer was not associated with physical performance or number of falls in geriatric 
Table 2. BP recovery and physical performance and falls

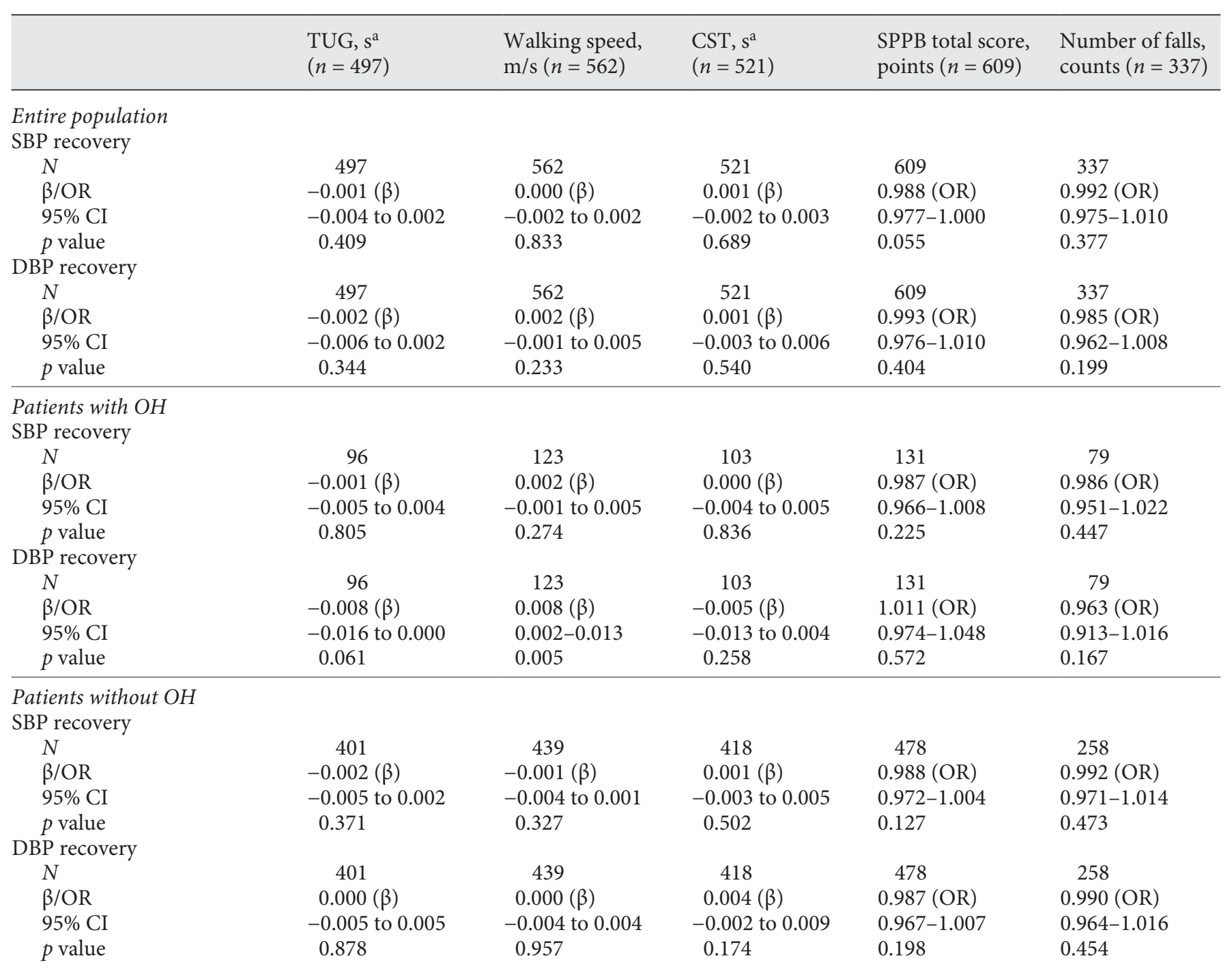

The table lists the results from the linear regression analyses with BP recovery as the independent variable, physical performance or falls as the dependent variable, and age and sex as covariates. SBP, systolic blood pressure; DBP, diastolic blood pressure; $\beta$, regression coefficient; OR, odds ratio; CI, confidence interval; TUG, timed up and go test; CST, chair stand test; SPPB, Short Physical Performance Battery. ${ }^{a}$ log-transformed.

outpatients. Orthostatic BP measurements using a sphygmomanometer have an inadequate time resolution to record the clinically relevant dynamics of orthostatic BP recovery. The results suggest that continuous BP measurements should be made routinely available and used in geriatric outpatient clinics to record clinically relevant dynamics of BP recovery. The results further suggest that the use of sphygmomanometer BP measurements should be avoided, despite their better availability. Further studies should point out whether continuously measured orthostatic BP recovery has potential to predict physical performance trajectories and falls. These studies should measure orthostatic BP recovery simultaneously using continuous BP measurement and a sphygmomanometer in the same population to better enable comparison of the clinical value of both techniques.

\section{Statement of Ethics}

The study was performed in accordance with the Declaration of Helsinki and approved by the local medical Ethics Committee of the VU University Medical Center Amsterdam (Center of Ge- 
riatrics Amsterdam cohort; ref. No. 2017.582) and the Institutional Review Board of the Leiden University Medical Center (ref. No. LUMC2010.x; Bronovo cohort), and patients gave written consent to participate in the study.

\section{Conflict of Interest Statement}

The authors have no conflicts of interest to declare.

\section{Funding Sources}

This study was supported by the perspective grant (NeuroCIMT, No. 14901) of the Applied and Engineering Sciences, which is part of the Netherlands Organization for Scientific Research (NWO, Utrecht, The Netherlands) and which is partly funded by the Ministry of Economic Affairs. Furthermore, this study was supported by the European Union's Horizon 2020 research and innovation program: PreventIT (No. 689238) and PANINI (No. 675003). The funders had no role in the study design, data collection and analysis, decision to publish, or preparation of the manuscript.

\section{Author Contributions}

Study concept and design: A. Mol, M.E.C. Blom, D.J. van den Bosch, C.G.M. Meskers, and A.B. Maier. Acquisition of data: M.E.C. Blom and D.J. van den Bosch. Analysis and interpretation of data: A. Mol, M.E.C. Blom, and D.J. van den Bosch. Drafting of the manuscript: A. Mol, M.E.C. Blom, and D.J. van den Bosch. Critical revision of the manuscript for important intellectual content: R.J.A. Van Wezel, C.G.M. Meskers, and A.B. Maier.

\section{References}

1 Freeman R, Wieling W, Axelrod FB, Benditt DG, Benarroch E, Biaggioni I, et al. Consensus statement on the definition of orthostatic hypotension, neurally mediated syncope and the postural tachycardia syndrome. Auton Neurosci. 2011;161(1-2):46-8.

2 Freeman R, Abuzinadah AR, Gibbons C, Jones P, Miglis MG, Sinn DI. Orthostatic hypotension: JACC state-of-the-art review. J Am Coll Cardiol. 2018;72(11):1294-309.

3 Mol A, Reijnierse EM, Bui Hoang PTS, van Wezel RJA, Meskers CGM, Maier AB. Orthostatic hypotension and physical functioning in older adults: a systematic review and metaanalysis. Ageing Res Rev. 2018 Dec;48:12244.

4 Mol A, Bui Hoang PTS, Sharmin S, Reijnierse EM, van Wezel RJA, Meskers CGM, et al. Orthostatic hypotension and falls in older adults: a systematic review and meta-analysis. J Am Med Dir Assoc. 2019 May;20(5):589-97.e5.

5 Iseli R, Nguyen VTV, Sharmin S, Reijnierse EM, Lim WK, Maier AB. Orthostatic hypotension and cognition in older adults: a systematic review and meta-analysis. Exp Gerontol. 2019;120:40-9.

6 Frith J, Bashir AS, Newton JL. The duration of the orthostatic blood pressure drop is predictive of death. Qjm. 2016;109(4):231-5.

7 Verwoert GC, Mattace-Raso FU, Hofman A, Heeringa J, Stricker BH, Breteler MM, et al. Orthostatic hypotension and risk of cardiovascular disease in elderly people: the Rotterdam study. J Am Geriatr Soc. 2008;56(10): 1816-20.
8 Finucane C, O'Connell MD, Fan CW, Savva GM, Soraghan CJ, Nolan H, et al. Age-related normative changes in phasic orthostatic blood pressure in a large population study: findings from The Irish Longitudinal Study on Ageing (TILDA). Circulation. 2014 Nov; 130(20):1780-9.

9 Finucane C, O'Connell MD, Donoghue O, Richardson K, Savva GM, Kenny RA. Impaired orthostatic blood pressure recovery is associated with unexplained and injurious falls. J Am Geriatr Soc. 2017 Mar;65(3):47482.

10 de Heus RAA, de Jong DLK, Rijpma A, Lawlor BA, Olde Rikkert MGM, Claassen JAHR Orthostatic blood pressure recovery is associated with the rate of cognitive decline and mortality in clinical Alzheimer's disease. J Gerontol A Biol Sci Med Sci. 2020 May; 75(11):2169-76.

11 Mol A, Slangen LRN, van Wezel RJA, Maier AB, Meskers CGM. Orthostatic blood pressure recovery associates with physical performance, frailty and number of falls in geriatric outpatients. J Hypertens. 2020 Aug;39:101-6.

12 Romero-Ortuno R, Cogan L, Foran T, Kenny RA, Fan CW. Continuous noninvasive orthostatic blood pressure measurements and their relationship with orthostatic intolerance, falls, and frailty in older people. J Am Geriatr Soc. 2011 Apr;59(4):655-65.

13 Romero-Ortuno R, O'Connell MD, Finucane C, Soraghan C, Fan CW, Kenny RA. Insights into the clinical management of the syndrome of supine hypertension - orthostatic hypotension ( $\mathrm{SH}-\mathrm{OH})$ : The Irish Longitudinal Study on Ageing (TILDA). BMC Geriatr. 2013 Dec; 13(1):73.
14 Lagro J, Schoon Y, Heerts I, Meel-van den Abeelen AS, Schalk B, Wieling W, et al. Impaired systolic blood pressure recovery directly after standing predicts mortality in older falls clinic patients. J Gerontol A Biol Sci Med Sci. 2014 Apr;69(4):471-8.

15 Juraschek SP, Daya N, Rawlings AM, Appel LJ, Miller ER, Windham BG, et al. Association of history of dizziness and long-term adverse outcomes with early vs. later orthostatic hypotension assessment times in middle-aged adults. JAMA Intern Med. 2017 Sep;177(9): 1316-23.

16 Juraschek SP, Lipsitz LA, Beach JL, Mukamal KJ. Association of orthostatic hypotension timing with clinical events in adults with diabetes and hypertension: results from the ACCORD trial. Am J Hypertens. 2019 Jun;32(7): 684-94.

17 Podsiadlo D, Richardson S. The timed "Up \& Go": a test of basic functional mobility for frail elderly persons. J Am Geriatr Soc. 1991 Feb; 39(2):142-8.

18 Guralnik JM, Simonsick EM, Ferrucci L, Glynn RJ, Berkman LF, Blazer DG, et al. A short physical performance battery assessing lower extremity function: association with self-reported disability and prediction of mortality and nursing home admission. J Gerontol. 1994 Mar;49(2):M85-94.

19 van Beek AH, Claassen JA, Rikkert MG, Jansen RW. Cerebral autoregulation: an overview of current concepts and methodology with special focus on the elderly. J Cereb Blood Flow Metab. 2008 Jun;28(6):1071-85. 